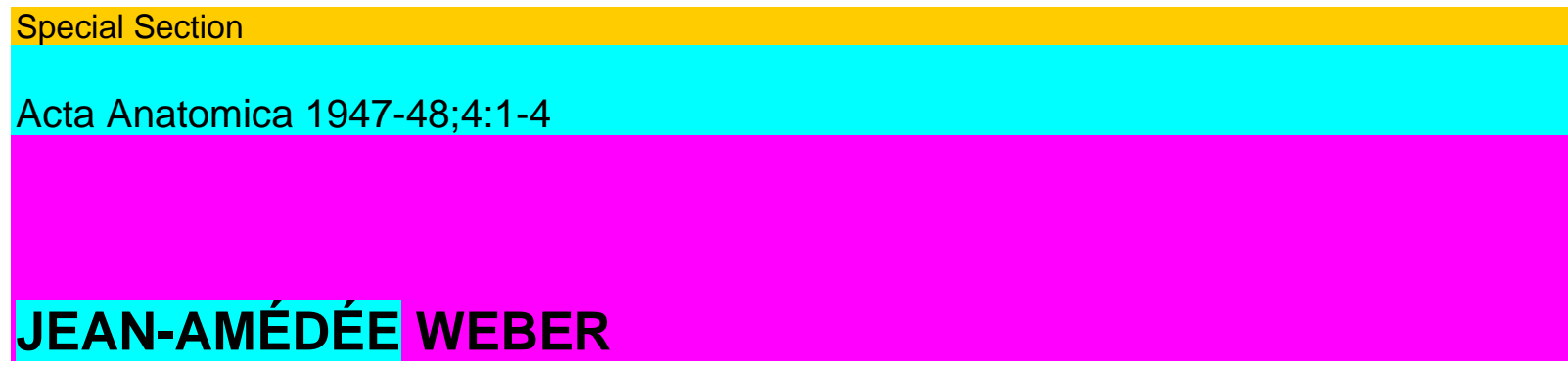

\title{
AU DOCTEUR
}

Â L'OCCASION DE SON

\section{0ÈME ANNIVERSAIRE ET DE SON JURILÉ PROFESSORAL} SEPTEMBRE 1947

J.-A. Weber, né à Mâcon (France) le 7 septembre 1877, Doc-teur en Médecine en 1903, Professeur agrégé d'Anatomie à la Faculté de Médecine de Nancy le 1 juillet 1904, Professeur d'Histologie et d'Embryologie puis d'Anatomie à la Faculté de Médecine d'Alger dès 1908, Professeur ordinaire d'Anatomie à l'Université de Geneve dès 1917, Professeur hono-raire dès 1947. Quelques amis ont voulu lui prouver ici leur estime. Quelques élèves le prient d'accepter ce modeste témoignage de reconnaissance et d'affection. Nombreux sont ceux qui lui présentent leurs felicitations et leurs voeux.

$1 *$

\section{INDEX}

Au Docteur Jean-Amédée "Weber 3

Phénomènes d "alteration des lerminaisons à la surface des cellules nerveuses dans un ganglion sym-

pathique chez Thomme. Par M. Barbey-Gampert-\&enève 5

Valeur, variations et equivalences des muscles extenseurs, interosseux, adducteurs et abducteurs de la

main et du pied, chez Thomme. Par J. A. Baumann-Qetiève

Recherches sur l'état biologique du boul central d'un rameau du nerf facial, sectionné expérime $\pi$ tale-

men!, dans diverses conditions, chez le cobaye. Par J. A. Baumann et A. Moret-Geneve . . 17

Observations sur le neurotropisme et le stéréotropisme dans la différenciation du système nerveux

central. Par W. v. Niederhäusern-Genève

Les tests et les facteurs extrïsèques et intri $\pi$ sèques du fonctionnement de la glande thyroïde. Par M. Aron et C. $\wedge$ 4ron-Strasbourg 27

Essai de thérapeutique neuro-chirurgicale des névralgies faciales centrales. Par M. Aubert et R.

Reyss-

Brion-L·yon

36

La structure inframiфroscopique des cylindraxes, étudiée par Гimprégnation argentique dïchroïtique. 
Anatomie, histologie et histophysiologie des glandes parathyroïdes du canard domestique. Par J. Benoit

et J. Cíaverí-Strasbourg

Absence des rotules et signification des os sésamoïdes. Par A. J. P, van den Broe\&-Utrecht (avec colla

boration du Dr. J. W.Barents)

Divisions nucléaires amitotiques dans des cultures de fibrocytes après administration de colchicine.

Par 0. Bucẁer-Zurich 60

L'ovaire du jeune cobaye, durant la période postnatale. Par E. Bujard-Genève 68

Les cellules sympathicotropes de Berger (avec Planche I). Par E. van Campenhout--LovГvaiti ... 73

Sur quelques faits cytologiques de Thistogénèse de la ré́tine. Par A. Celestíno da Cosía-Lisbonne .. 79

Le bulbe de Tinfundibulum diencéphalique chez le cobaye. Par R. Collin-ISancy

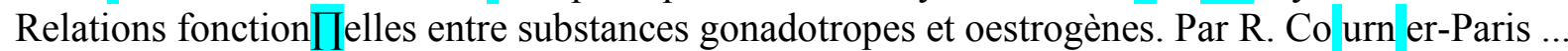
94

Sur les operations susceptibles de provoquer 1'inversion du situs viscerum chez les anoures. Par A. Dalcq-

Bruxelles 100

Lignes papillaires en cas de syndactylie Par J. Dankmeijer-L·eyàe et J. M. Waltman·La Haye . . . 108

A study of human splanchnic nerves removed at operation for hypertension. By H. C. B. DenberNew York 117

Übe $\Gamma$ die Variabilität der Arteriae thyreoideae. Von A. Faller und 0. Sc $7 / 8$ ärer-Fribourg 119

Degenerationsstudien über das optische Gefaiet im Hypothalamus des Meerschweinchens. Von

E. Frey-

Zurich 123

Sur la nature des cellules co $\pi$ tenues dans les ampoules dermiques de Plotosus anguillaris. Par P.

Gerard'

Bruxelles 137

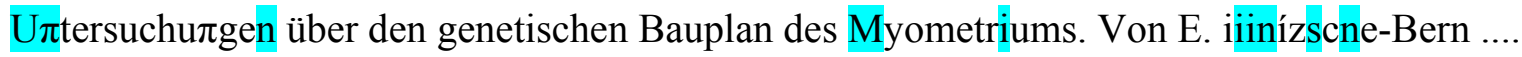
142

La formation du cristalli $\pi$ et la determination de Гcbauche cristallinienne. Par J. Jolly-Paris . . . 149

Les vaisseaux sanguins de la prostate. Par F. .Kiss-Budapest

Sur des problèmes poses par la cicatrisation des nerfs chez Thomme. Par M. iПleira-Strasbourg. . 161

Le neuroépithélïum olfactif. Par H. Lams-Gand 168

i $>$ e quelques problèmes histologiques poses par la chirurgie physiologique contemporaine. Par

R. Leríche-

Paris

Le processus de division mitotique dans les cellules étalées en surface. Par G. Levi-Turin 176 
L'aponévrose dorsale moyenne de la main. Par M. J1/8cien-Nancy

Über die Anlage der Nachniere bei der Maus. Von E. Litc/icig-Basel

La massa intermedia ou commissure grise. Par F. Morel-Geneve

Contribution à $\Gamma$ étude de Torigine de $\Gamma$ entoblaste chez les inßecles. Par J. MuZnord-Bruxelles . . 208

Structure de la membrane keratinoïde de $\Gamma \mathrm{e}^{5} / 8$ tomac musculaire de Gallus gallus. Par 0. M.

Olivo-

Bologne

213

Sur la localisation du pronéphros des anoures. Par J. Pasíeeïs-Bruxelles

Sur la croissance des, cultures de fibroblastes dans un champ magnétique. Par N. Perak is -

Strasbourg 225

La pcriphérie du lobule pulmonaire chez Thomme. Par A. Policard-L·yo $\tau$ i

Cyclopie par Гaction de Lithium chez la Limnée. Par Chr. P. /føfen-Utrecht

Breves remarques sur quelques points de la pathologic du tissu conjonctif. Par F. Roulet-Jiåle (avec

Planche II) 248

Action de la colchicine et de $\Gamma$ hydrate de chloral sur Гoeuf de Triturus helveticus L. en développement.

Par P. SeníPÌn-Montpellier 256

Zur Wirkung des StUboestrols auf die Furchungsteilunge $\pi$ des Axolotleies. Von G. TóWuryZürLch269

Etude comparative de reactions nucléales. Par J. TurcÁirat-Montpellier

Sur Taccoutumance aux toxiques des cellules cultivécs in vitro. Par J. Feme-Paris 282

Quelques essais de chirurgie sympathique dans les troubles sudoraux. Par P. Wertaeimer et J. Barrie-

Lyon 288

Die funktionelle Struktur der Ligamenta anularia der menschlichen Trachea. Von G. WolfHeidegger-Basel 295

Zur Histologie der menschlichen Unterkieferdrüse. Von if. $Z_{i}$ eg/er-Bern (mit Tafel III) 